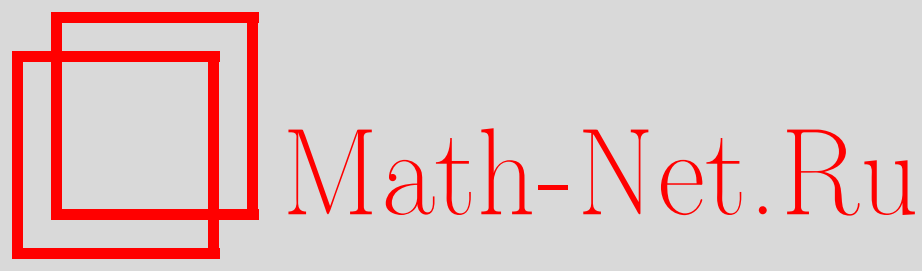

Р. А. Зубов, Е. В. Прохватилов, М. Ю. Малышев, Модель кварк-антикваркового взаимодействия в квантовой хромодинамике на световом фронте, ТМФ, 2017, том 190, номер 3, 440-454

DOI: https://doi.org/10.4213/tmf9133

Использование Общероссийского математического портала Math-Net.Ru подразумевает, что вы прочитали и согласны с пользовательским соглашением http: //www . mathnet.ru/rus/agreement

Параметры загрузки:

IP : 34.229 .108 .108

26 апреля 2023 г., 11:20:32

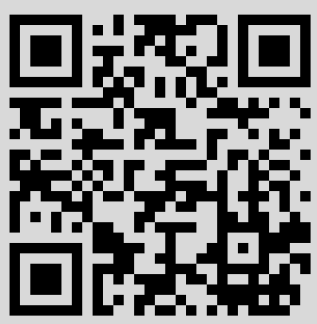




\title{
ФИЗИКА
}

Том 190, № 3

март, 2017

(C) 2017 г. Р. А. Зубов*, Е. В. Прохватилов*, М. Ю. Малышев ${ }^{\dagger *}$

\section{МОДЕЛЬ КВАРК-АНТИКВАРКОВОГО ВЗАИМОДЕЙСТВИЯ В КВАНТОВОЙ ХРОМОДИНАМИКЕ НА СВЕТОВОМ ФРОНТЕ}

\begin{abstract}
Сформулирована модель кварк-антикваркового взаимодействия, связанная с рассмотрением предельного перехода к гамильтониану на световом фронте в квантовой хромодинамике. В качестве ультрафиолетовой регуляризации используется решетка в пространстве поперечных координат. Кроме того, вводятся ограничение продольной координаты светового фронта и периодические граничные условия. Нулевая мода поля по этой координате рассматривается как независимая динамическая переменная. Пространство состояний модели ограничивается кварком и антикварком, которые взаимодействуют только посредством нулевой моды глюонного поля на световом фронте. В рамках этого подхода получен дискретный спектр масс связанных состояний. Этот спектр определяется уравнением, которое по продольной координате оказывается аналогичным уравнению 'т Хоофта двумерной квантовой хромодинамики. Данное уравнение также содержит кварк-антикварковый потенциал, который обеспечивает конфайнмент в поперечном пространстве.
\end{abstract}

Ключевые слова: гамильтонов подход, квантовая хромодинамика, спектр масс, световой фронт, уравнение 'т Хоофта, кварк-антикварковая модель.

DOI: https://doi.org/10.4213/tmf9133

\section{1. ВВЕДЕНИЕ}

Настоящая работа является продолжением исследования возможности построить эффективную модель взаимодействия кварков и антикварков в рамках подхода, связанного с квантованием на световом фронте (СФ) [1]. СФ определяется уравнением $x^{+}=0$, где $x^{+}$играет роль времени в координатах СФ [2]

$$
x^{ \pm}=\frac{x^{0} \pm x^{3}}{\sqrt{2}}, \quad x^{\perp}=\left(x^{1}, x^{2}\right) .
$$

Работа выполнена при поддержке Санкт-Петербургского государственного университета (грант № 11.38.189.2014).

*Санкт-Петербургский государственный университет, Санкт-Петербург, Россия. E-mail: roman.zubov@hep.phys.spbu.ru,e.prokhvatilov@spbu.ru,m.malyshev@spbu.ru

${ }^{\dagger}$ Петербургский институт ядерной физики им. Б. П. Константинова, Национальный исследовательский центр "Курчатовский институт", Гатчина, Ленинградская обл., Россия 
Здесь $x^{0}, x^{3}, x^{\perp}$ - это обычные лоренцевы координаты. Квантование на СФ широко применяется для описания процессов рассеяния частиц при высоких энергиях, когда импульсы частиц велики, а скорость имеет порядок скорости света. В этом случае в квантовой хромодинамике (КХД) применима теория возмущений по константе взаимодействия. В рамках такой теории возмущений при квантовании на СФ обычно используется регуляризация $\left|p_{-}\right| \geqslant \varepsilon>0, p_{ \pm}=\left(p_{0} \pm p_{3}\right) / \sqrt{2}$, которая отвечает тому, что пренебрегают модами полей по продольной координате СФ, близкими к нулевой моде $p_{-}=0$. Отбрасывание этих мод позволяет регуляризовать особенности, связанные с квантованием на СФ, но, возможно, порождает отличия теории возмущений на СФ от обычной теории возмущений при квантовании на поверхности постоянного времени в лоренцевых координатах [3]-[6]. Единственный найденный способ устранения таких отличий в КХД в калибровке СФ - это введение дополнительных “духовых" полей, аналогичных используемым при регуляризации Паули-Вилларса [7].

Применение квантования на СФ в области низких и промежуточных энергий, например для описания связанных состояний полей в КХД, основано на попытках решать непертурбативную задачу на собственные значения гамильтониана на СФ в пространстве Фока с "простым" физическим вакуумом. Этот вакуум определяется как состояние, отвечающее низшему собственному значению оператора импульса $P_{-} \geqslant 0$. При сохранении лоренц-инвариантности это состояние отвечает также минимуму оператора $P_{+}$. Регуляризация $\left|p_{-}\right| \geqslant \varepsilon$ нарушает эту лоренцеву симметрию. Кроме того, окрестность нулевых мод $\left|p_{-}\right|<\varepsilon$ может оказаться существенной для непертурбативной области низких энергий (например, возникают трудности с описанием вакуумных конденсатов [8]).

Другая возможная регуляризация, а именно ограничение пространства по продольной координате СФ, $\left|x^{-}\right| \leqslant L$, с периодическими граничными условиями для полей, дискретизует импульс как $p_{-}=p_{n}=\pi n / L$ и позволяет ввести нулевую моду $\left(p_{-}=n=0\right)$, четко отделив ее от ненулевых $(n=1,2, \ldots)$. Однако в этом случае нулевая мода не является независимой динамической переменной и должна быть выражена через ненулевые моды путем решения сложных и плохо определенных уравнений связей [9], [10].

Следующая попытка была связана с исследованием предельного перехода к гамильтониану на СФ от теории, квантованной на пространственноподобной плоскости, приближающейся к СФ [6], [8], [11], [12]. На примере квантовой электродинамики (КЭД) в 1+1 измерениях (т. е. массивной модели Швингера) была обнаружена зависимость результата от способа предельного перехода [8]. Например, если переходить на СФ, сохраняя условие $\left|x^{-}\right| \leqslant L$, то становится невозможным описать фермионный конденсат, характерный для данной модели. Однако приближенно конденсат можно учесть, если рассматривать предел $L \rightarrow \infty$ одновременно с предельным переходом на СФ. К тому же в модели $(1+1)$-мерной КЭД оказывается возможной [13], [8] корректная формулировка на СФ, если нулевая мода становится независимой динамической переменной после надлежащей модификации теории, т. е. лагранжиана и гамильтониана. С точки зрения предельного перехода на СФ такую модификацию можно ввести следующим образом. Сначала фиксируем $\left|x^{-}\right| \leqslant L$ и совершаем переход к гамильтониану на СФ при сохранении членов гамильтониана, содержащих только нулевые моды, в виде, соответствующем теории на поверхности, 
близкой к СФ, т. е. “замораживая" для них предельный переход на СФ. Полученный таким образом эффективный гамильтониан позволяет в $(1+1)$-мерной КЭД вычислять в пределе $L \rightarrow \infty$ как спектр масс, так и конденсат при надлежащем выборе параметра $a_{0}=L \eta_{0}$. Этот параметр связывает величину $L$ и угол $\eta_{0}^{2} / 2$ отклонения от СФ плоскости, на которой определены “замороженные" члены гамильтониана с нулевыми модами. В этом случае предел $L \rightarrow \infty$ соответствует $\eta_{0} \rightarrow 0$.

Для КХД в $3+1$ измерениях не существует точных решений в области сильной связи, т. е. области, характерной для описания спектра адронов. Поэтому указанные выше способы введения динамической нулевой моды могут иметь только полуфеноменологический характер. Кроме того, в $3+1$ измерениях появляются поперечные координаты и связанные с ними компоненты калибровочного поля в КХД. В калибровке СФ эти компоненты описывают динамику калибровочного поля. Если в качестве регуляризации по поперечным импульсам ввести решетку в поперечном пространстве, то можно сохранить калибровочную инвариантность относительно преобразований, сохраняющих калибровку СФ. Для этого достаточно отнести нулевые моды поперечных компонент глюонного поля к ребрам решетки и сопоставить им унитарные матрицы $U_{\perp}$, а ненулевые моды отнести к узлам и описывать их эрмитовыми матрицами [14]. Тогда возможно совершить предельный переход к эффективному гамильтониану на СФ при сохранении условия $\left|x^{-}\right| \leqslant L$, "замораживая" динамику нулевой моды в том виде, в каком она присутствует в гамильтониане теории вблизи СФ. Формулировка теории вблизи СФ связана с введением координат $y^{\mu}$, близких к координатам СФ, и с квантованием на пространственноподобной поверхности $y^{0}=0$ [8], [11]:

$$
y^{0}=x^{+}+\frac{\eta^{2}}{2} x^{-}, \quad y^{3}=x^{-}, \quad y^{\perp}=x^{\perp} .
$$

Заметим, что в пределе $\eta \rightarrow 0$ они совпадают с координатами СФ. Соответствующие этим координатам импульсы $q_{\mu}$ имеют вид

$$
q_{0}=p_{+}, \quad q_{3}=p_{-}-\frac{\eta^{2}}{2} p_{+}, \quad q_{\perp}=p_{\perp} .
$$

В дальнейшем будем называть введенные таким образом координаты $\eta$-координатами. Геометрическая интерпретация параметра $\eta^{2} / 2$ (при малых $\eta$ ) как угла отклонения плоскости квантования от плоскости СФ приведена на рис. 1.

Различие предельных переходов для нулевых и ненулевых мод существенно нарушает лоренцеву симметрию и делает неоднозначным выбор выражения для оператора $M^{2}$ квадрата массы. В релятивистски-инвариантной теории оно имеет вид $M^{2}=2 P_{+} P_{-}-P_{\perp}^{2}$ в координатах СФ или $M^{2}=2 Q_{3} Q_{0}+\eta^{2} Q_{0}^{2}-P_{\perp}^{2}$ в $\eta$-координатах. Для нулевой моды можно использовать вышеприведенное выражение в $\eta$ координатах, полагая в нем $q_{3}=0$. При этом в качестве $Q_{0}$ можно взять ту часть эффективного гамильтониана, которая содержит только нулевые моды, вычитая из нее вакуумное значение [14]. Обозначим этот вклад через $H_{(0)}$. Для оставшейся части эффективного гамильтониана, обозначаемой как $H_{(\emptyset)}$, можно использовать выражение для $M^{2}$ в координатах СФ, отождествляя $P_{+}$с $H_{(\emptyset)}$. Простейший выбор оператора $M^{2}$, который в пределе $L \rightarrow \infty$ ведет к восстановлению лоренцинвариантности и согласуется с условием $L \eta_{0}=$ const, соответствует сумме двух 


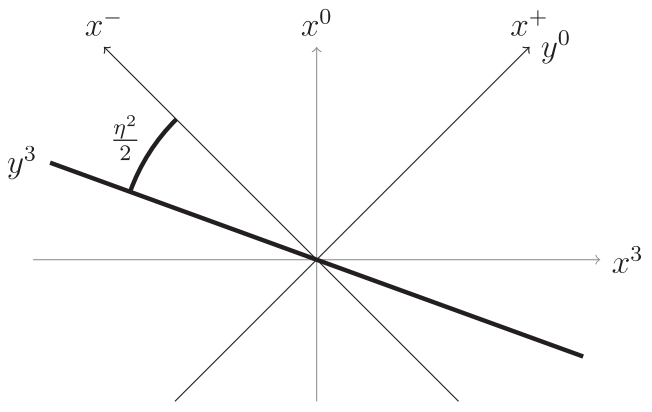

Рис. 1. Геометрическая интерпретация параметра $\eta^{2} / 2$ (при малых $\eta$ ) как угла отклонения плоскости квантования нулевой моды от плоскости СФ.

вышеуказанных вкладов:

$$
M_{\mathrm{eff}}^{2}=2 P_{-} H_{(\emptyset)}+\eta_{0}^{2} H_{(0)}^{2}-P_{\perp}^{2} .
$$

Данный вариант выбора выражения для $M^{2}$ оставляет спектр масс конечным при $L \rightarrow \infty, L \eta_{0}=$ const.

Чтобы опробовать полученную модель, мы сначала рассматриваем $(2+1)$-мерную КХД и сужаем пространство состояний до кварка и антикварка, взаимодействующих с поперечным глюонным полем только посредством его нулевой моды. Кроме этого мы модифицируем член в гамильтониане, который порождается решением калибровочной связи и имеет вид четырехфермионного оператора, нелокального по координате $x^{-}$, но локального по $x^{\perp}$. Мы делаем этот член нелокальным по $x^{\perp}$, раздвигая входящие в него фермионные токи и усредняя по области порядка размера адрона $L_{\text {had }}$. Это необходимо для того, чтобы данный оператор давал ненулевой вклад на состояниях с ненулевой длиной в поперечном пространстве.

Вычисление спектра оператора $M^{2}$ сводится к вычислению его собственных значений на пространстве состояний кварка и антикварка, соединенных в поперечном направлении "струной” из реберных переменных. В пределе снятия регуляризации эта задача становится аналогичной решению уравнения, похожего на уравнение 'т Хоофта [15] по $x^{-}$и включающего потенциал, квадратичный по поперечному расстоянию между кварком и антикварком. При этом достаточно перенормировать только константу связи, если считать параметр $a_{0}=L \eta_{0}$, как и размер нелокальности по $x^{\perp}$, величинами порядка размера адрона $L_{\mathrm{had}}$. Заметим, что формула, определяющая перенормировку константы связи, имеет другой вид, нежели в обычной теории возмущений.

Следующий шаг - построение аналогичной модели для КХД в $3+1$ измерениях может быть сделан повторением шагов, которые были предприняты в $2+1$ измерениях. Однако переход к двумерной решетке в поперечном пространстве значительно усложняет анализ. Предполагая, что кварк и антикварк соединены “калибровочной струной" преимущественно по кратчайшему пути в поперечной плоскости, можно прийти к уравнению, являющемуся простым обобщением уравнения для спектра масс в $(2+1)$-мерном случае. В настоящей работе вычисляется спектр этого уравнения и обсуждается возможность его сравнения с экспериментальным спектром мезонов с присущим ему вырождением по квантовым числам. 


\section{2. КВАРК-АНТИКВАРКОВАЯ МОДЕЛЬ}

Рассмотрим сначала модель взаимодействия кварка и антикварка в $2+1$ измерениях для $S U(N)$-калибровочно-инвариантной теории [1]. В этой модели решетка в поперечном пространстве (с параметром $a$ ) одномерна и имеет конечное число узлов $K_{\perp}$. Для глюонных и кварковых полей вводятся периодические граничные условия на интервале $0 \leqslant x_{\perp} \leqslant K_{\perp} a$. В дальнейшем для краткости координата $x^{\perp}$ обозначается символом $x$ и подразумевается зависимость всех полей от координаты $x^{-}$там, где необходимо. Пространство Фока на СФ сужается таким образом, чтобы существовали только состояния с одной кварк-антикварковой парой. Также считается, что фермионы взаимодействуют только через нулевые моды поперечных компонент глюонного поля $U_{\perp} \equiv U$, а операторы рождения ненулевых мод калибровочного поля отсутствуют. Гамильтониан этой модели с массой кварка $m_{\mathrm{q}}$ и безразмерной константой связи $g^{2} a$ записывается в виде [1], [14]

$$
\begin{array}{rl}
H=\sum_{x} \int_{-L}^{L} & d x^{-}\left\{\frac{g^{2} a}{8 L^{2} \eta_{0}^{2}} \pi^{a}(x) \pi^{a}(x)+\frac{g^{2} a}{2} \partial_{-}^{-1}\left(\chi^{\dagger}(x) \frac{\lambda^{a}}{2} \chi(x)\right) \partial_{-}^{-1}\left(\chi^{\dagger}(x) \frac{\lambda^{a}}{2} \chi(x)\right)-\right. \\
- & \frac{i}{8 a}\left[\chi^{\dagger}(x-a) \sigma_{1} U^{\dagger}(x)-\chi^{\dagger}(x+a) U(x+a) \sigma_{1}+2 m_{\mathrm{q}} a \chi^{\dagger}(x)\right] \times \\
& \times \partial_{-}^{-1}\left[U(x) \sigma_{1} \chi(x-a)-\sigma_{1} U^{\dagger}(x+a) \chi(x+a)+2 m_{\mathrm{q}} a \chi(x)\right]+ \\
& \left.+\frac{g^{2} a}{2} \frac{1}{4 a^{2} L^{2}} \frac{1}{2}\left(N-\frac{1}{N}\right) \sum_{\substack{m \in \mathbb{Z}+1 / 2 \\
m>0}}\left(b_{m}^{\dagger}(x) b_{m}(x)+d_{m}^{\dagger}(x) d_{m}(x)\right) s_{m}\right\}
\end{array}
$$

где введено обозначение

$$
s_{m}=\sum_{\substack{k \in \mathbb{Z}+1 / 2, k>0, k \neq m}}^{\infty}\left[\frac{1}{\left(p_{m}-p_{k}\right)^{2}}-\frac{1}{\left(p_{m}+p_{k}\right)^{2}}\right]
$$

с дискретизованным продольным импульсом $p_{m}=\pi m / L$. Фермионные поля $\chi(x)$ выражаются через операторы рождения и уничтожения следующим образом:

$$
\chi_{r}^{i}(x)=\frac{1}{\sqrt{2 a L}} \sum_{\substack{m \in \mathbb{Z}+1 / 2, m>0}}\left(b_{m r}^{i}(x) e^{-i p_{m} x^{-}}+d_{m r}^{i \dagger}(x) e^{i p_{m} x^{-}}\right),
$$

где $r= \pm 1 / 2$ - спиновый индекс, а $i=1,2, \ldots, N$ суть индексы присоединенного представления группы $S U(N)$ со структурными константами $f^{a b c}$. В этих выражениях $\sigma_{k}$ и $\lambda^{a}$ обозначают матрицы Паули и Гелл-Манна соответственно. Переменные $\pi^{a}(x)$ и $U(x)$ удовлетворяют коммутационным соотношениям (при $x^{+}=x^{\prime+}=0$ )

$$
\left[\pi^{a}(x), U\left(x^{\prime}\right)\right]=-\delta_{x x^{\prime}} \frac{\lambda^{a}}{2} U(x), \quad\left[\pi^{a}(x), \pi^{b}\left(x^{\prime}\right)\right]=i \delta_{x x^{\prime}} f^{a b c} \pi^{c}(x),
$$


а операторы рождения кварка и антикварка $b_{m}^{\dagger}, d_{m}^{\dagger}-$ соответствующим антикоммутационным соотношениям (при $x^{+}=x^{++}=0$ )

$$
\left\{b_{m r}^{i}(x), b_{m^{\prime} r^{\prime}}^{i^{\prime} \dagger}\left(x^{\prime}\right)\right\}=\left\{d_{m r}^{i}(x), d_{m^{\prime} r^{\prime}}^{i^{\prime} \dagger}\left(x^{\prime}\right)\right\}=\delta_{m m^{\prime}} \delta_{x x^{\prime}} \delta_{i i^{\prime}} \delta_{r r^{\prime}} .
$$

Также здесь использовано обозначение $\mathbb{Z}+1 / 2$ для множества полуцелых чисел, т. е. $k=1 / 2,3 / 2, \ldots$.

В соответствии с нашим ограничением пространства Фока на СФ можно написать выражение для состояний, входящих в его базис:

$$
\left|\begin{array}{l}
l \\
m
\end{array}\right\rangle \equiv \frac{1}{\sqrt{N K_{\perp}}} \sum_{x} b_{m}^{\dagger}(x) U_{x, x+l a} d_{n-m}^{\dagger}(x+l a)|0\rangle, \quad\left\langle l_{m_{2}} \mid{ }_{m_{2}}^{l_{1}}\right\rangle=\delta_{m_{2} m_{1}} \delta_{l_{2} l_{1}},
$$

где $l$ - это “длина" цепочки базисного состояния в единицах $a,-K_{\perp} \leqslant l \leqslant K_{\perp}$, и использовано обозначение

$$
U_{x, x^{\prime}} \equiv \begin{cases}U^{\dagger}(x+a) U^{\dagger}(x+2 a) \ldots U^{\dagger}\left(x^{\prime}\right), & \text { если } x<x^{\prime}, \\ U(x) U(x-a) \ldots U\left(x^{\prime}+a\right), & \text { если } x>x^{\prime}, \\ I, & \text { если } x=x^{\prime} .\end{cases}
$$

Полный продольный импульс такой кварк-антикварковой системы есть

$$
p_{-} \equiv p_{n}=\frac{\pi n}{L} .
$$

Простое суммирование по всем узлам решетки в определении (10) базиса $\left|\begin{array}{c}l \\ m\end{array}\right\rangle$ обеспечивает трансляционную инвариантность любого состояния, что в свою очередь соответствует поперечному импульсу $p_{\perp}=0$. Поскольку согласно работе [14] закон преобразования матриц $U$ при калибровочном преобразовании $\Omega(x)$ имеет вид

$$
\Omega(x) U(x) \Omega^{\dagger}(x-a),
$$

введение в определение базисных состояний цепочки из матриц $U$ делает эти состояния инвариантными относительно калибровочных преобразований, зависящих только от $x^{\perp}$.

Четырехфермионный оператор

$$
\partial_{-}^{-1}\left(\chi^{\dagger}(x) \frac{\lambda^{a}}{2} \chi(x)\right) \partial_{-}^{-1}\left(\chi^{\dagger}(x) \frac{\lambda^{a}}{2} \chi(x)\right)
$$

в гамильтониане (5) после нелокальной модификации [1] выглядит следующим образом:

$$
\begin{aligned}
\frac{a}{2 L_{\mathrm{had}}} \sum_{l=-L_{\mathrm{had}} / a}^{L_{\mathrm{had}} / a} \partial_{-}^{-1}\left(\chi^{\dagger}(x) U_{x, x^{\prime}} \frac{\lambda^{a}}{2} U_{x^{\prime}, x} \chi(x)\right) \times \\
\quad \times \partial_{-}^{-1}\left(\chi^{\dagger}(x+l a) U_{x+l a, x^{\prime}} \frac{\lambda^{a}}{2} U_{x^{\prime}, x+l a} \chi(x+l a)\right),
\end{aligned}
$$

где $x^{\prime}$ - это произвольная точка поперечного пространства. Такая модификация позволяет расширить действие этого оператора на состояния с ненулевой длиной в поперечном пространстве. 
Как уже упомянуто во введении, в качестве выражения для квадрата массы выбирается выражение (при $p_{\perp}=0$ и $p_{-}=p_{n}$ )

$$
M_{\mathrm{eff}}^{2}=2 p_{n} H_{(\emptyset)}+\eta_{0}^{2} H_{(0)}^{2},
$$

где $H_{(0)}$ включает в себя член $\pi^{a} \pi^{a}$ из $(5)$, а $H_{(\varnothing)}$ - все прочие члены. На рассматриваемых нами состояниях спектр такого оператора $M^{2}$ оказывается конечным при $L \rightarrow \infty, L \eta_{0}=$ const при соответствующем выборе перенормировки константы связи $g$. При этом феноменологический параметр $L \eta_{0}$ следует считать величиной порядка размера адрона, т. е.

$$
L \eta_{0}=\frac{L_{\mathrm{had}}}{\alpha}
$$

с некоторым параметром $\alpha \sim 1$. Тогда указанная перенормировка для безразмерной константы связи $g^{2} a$ имеет вид

$$
g^{2} a=\bar{g}^{2} \frac{a}{L_{\mathrm{had}}},
$$

где $\bar{g}$ - это эффективная безразмерная константа связи на масштабе $L_{\text {had }}$.

Теперь в терминах безразмерных переменных

$$
\lambda=M^{2} L_{\mathrm{had}}^{2}, \quad r=\frac{l a}{L_{\mathrm{had}}}, \quad \bar{m}_{\mathrm{q}}=m_{\mathrm{q}} L_{\mathrm{had}}, \quad \xi=\frac{p_{m}}{p_{n}}
$$

можно получить уравнение для спектра связанных состояний в этой модели в пределе снятия регуляризации $L \rightarrow \infty, L \eta_{0}=$ const, $a \rightarrow 0$ :

$$
\begin{gathered}
\lambda f(\xi, r)=\left[\left(\frac{\alpha \bar{g}^{2}}{4} \frac{1}{2}\left(N-\frac{1}{N}\right)\right)^{2} r^{2}+\left(\frac{1}{\xi}+\frac{1}{1-\xi}\right)\left(-\frac{\partial^{2}}{\partial r^{2}}+\bar{m}_{\mathrm{q}}^{2}\right)\right] f(\xi, r)- \\
-\frac{\bar{g}^{2}}{2 \pi} \frac{1}{2}\left(N-\frac{1}{N}\right) \mathrm{P} \int_{0}^{1} d \xi^{\prime} \frac{f\left(\xi^{\prime}, r\right)-f(\xi, r)}{\left(\xi^{\prime}-\xi\right)^{2}}
\end{gathered}
$$

где $f(\xi, r)$ - это волновая функция связанного состояния кварка и антикварка, а интеграл понимается в смысле главного значения. Переменная $\xi$ становится в этом пределе непрерывной и принимает значения из интервала $0 \leqslant \xi \leqslant 1$. Заметим также, что часть этого уравнения, связанная с переменной $\xi$, имеет вид уравнения 'т Хоофта [15] в двумерной КХД.

\section{3. МОДЕЛЬ В $3+1$ ИЗМЕРЕНИЯХ}

Физический интерес представляет построение аналогичной модели для $(3+1)-$ мерной КХД. Соответствующий эффективный гамильтониан на СФ был получен в работе [14]. Чтобы перейти к нашей кварк-антикварковой модели, рассмотрим проекцию этого гамильтониана на пространство Фока, суженное аналогично $(2+1)$ - 
мерному случаю. Эта проекция имеет следующий вид:

$$
\begin{aligned}
H= & \sum_{x} \int_{-L}^{L} d x^{-}\left\{\frac{g^{2}}{8 L^{2} \eta_{0}^{2}} \sum_{k=1}^{2} \pi_{k}^{a}(x) \pi_{k}^{a}(x)+\right. \\
& +\frac{g^{2} a^{2}}{2} \partial_{-}^{-1}\left(\chi^{\dagger}(x) \frac{\lambda^{a}}{2} \chi(x)\right) \partial_{-}^{-1}\left(\chi^{\dagger}(x) \frac{\lambda^{a}}{2} \chi(x)\right)- \\
& -\frac{i}{8} \sum_{k^{\prime}=1}^{2} \sum_{k=1}^{2}\left[\chi^{\dagger}\left(x-a_{k^{\prime}}\right) \sigma_{k^{\prime}} U_{k^{\prime}}^{\dagger}(x)-\chi^{\dagger}\left(x+a_{k^{\prime}}\right) U_{k^{\prime}}\left(x+a_{k^{\prime}}\right) \sigma_{k^{\prime}}+2 m_{\mathrm{q}} a \chi^{\dagger}(x)\right] \times \\
& \times \partial_{-}^{-1}\left[U_{k}(x) \sigma_{k} \chi\left(x-a_{k}\right)-\sigma_{k} U_{k}^{\dagger}\left(x+a_{k}\right) \chi\left(x+a_{k}\right)+2 m_{\mathrm{q}} a \chi(x)\right]+ \\
& \left.+\frac{g^{2} a^{2}}{2} \frac{1}{4 a^{4} L^{2}} \frac{1}{2}\left(N-\frac{1}{N}\right) \sum_{\substack{m \in \mathbb{Z}+1 / 2 \\
m>0}}\left(b_{m}^{\dagger}(x) b_{m}(x)+d_{m}^{\dagger}(x) d_{m}(x)\right) s_{m}\right\},
\end{aligned}
$$

где фермионные поля $\chi(x)$ определяются выражением

$$
\chi_{r}^{i}(x)=\frac{1}{a \sqrt{2 L}} \sum_{\substack{m \in \mathbb{Z}+1 / 2, m>0}}\left(b_{m r}^{i}(x) e^{-i p_{m} x^{-}}+d_{m r}^{i \dagger}(x) e^{i p_{m} x^{-}}\right) .
$$

Здесь мы по-прежнему для обозначения поперечных координат $x^{\perp}=\left(x^{1}, x^{2}\right)$ используем символ $x$ и подразумеваем зависимость всех полей от координаты $x^{-}$там, где необходимо. Символ $a_{k}$ обозначает вектор в направлении $x^{k}$ с длиной, равной шагу решетки $a$.

Выражение $(21)$ отличается от $(2+1)$-мерного случая $(5)$ суммированием по всем узлам $x^{\perp}$ двумерной $\left(K_{\perp} \times K_{\perp}\right)$-решетки и присутствием двух поперечных компонент $U_{1}$ и $U_{2}$ нулевой моды глюонного поля. Также в нем присутствует дополнительный (не выписанный в этом уравнении) член $\operatorname{tr}\left(G_{12}^{\dagger} G_{12}\right)$, где $G_{12}$ является решеточным аналогом соответствующей компоненты тензора напряженности глюонного поля $F_{\mu \nu}$. В рассматриваемой модели этот член дает вклад только в вакуумное значение [14] эффективного гамильтониана и при нашем определении оператора квадрата массы $M^{2}$ не влияет на его спектр.

Базис пространства Фока на СФ этой модели можно записать теперь следующим образом:

$$
\begin{aligned}
\left|{ }_{m}^{\Delta x}, \mathcal{S}\right\rangle \equiv & \frac{1}{K_{\perp} \sqrt{N}} \sum_{x} b_{m}^{\dagger}(x) U_{x, x+\Delta x}^{\mathcal{S}} d_{n-m}^{\dagger}(x+\Delta x)|0\rangle, \\
& \left\langle\underset{m^{\prime}}{\Delta}, \mathcal{S}^{\prime} \mid{ }_{m}^{\Delta x}, \mathcal{S}\right\rangle=\delta_{m m^{\prime}} \delta_{l_{1} l_{1}^{\prime}} \delta_{l_{2} l_{2}^{\prime}},
\end{aligned}
$$

где $p_{n}=\pi n / L$ - полный импульс состояния, а $\Delta x=\left(l_{1} a, l_{2} a\right)$ - вектор смещения антикварка относительно кварка на двумерной поперечной решетке. Здесь через $U_{x, x^{\prime}}^{\mathcal{S}}$ обозначены цепочки из матриц $U_{k}^{\dagger}$ и $U_{k}(k=1,2)$, соединяющие точки $x$ и $x^{\prime}$ двумерного поперечного пространства по пути $\mathcal{S}$ и преобразующиеся так же, как матрицы $U_{\perp}$. Это означает, что в базисе присутствуют состояния, в которых кварк и антикварк связаны "калибровочной струной" по бесконечному числу возможных путей. Это сильно затрудняет анализ модели. Однако если предположить, что кварк и антикварк связаны между собой преимущественно по кратчайшему пути и, соответственно, сопоставить каждому их положению только одно состояние в базисе, то удается получить уравнение для спектра масс этой модели. 
В данном случае нелокальная модификация четырехфермионного члена в гамильтониане $(21)$ обобщается на $(3+1)$-мерный случай естественным образом:

$$
\begin{aligned}
\frac{a}{2 L_{\mathrm{had}}} \sum_{\Delta x} \partial_{-}^{-1}\left(\chi^{\dagger}(x) U_{x, x^{\prime}}^{\mathcal{S}} \frac{\lambda^{a}}{2} U_{x^{\prime}, x}^{\mathcal{S}} \chi(x)\right) \times \\
\quad \times \partial_{-}^{-1}\left(\chi^{\dagger}(x+\Delta x) U_{x+\Delta x, x^{\prime}}^{\mathcal{S}^{\prime}} \frac{\lambda^{a}}{2} U_{x^{\prime}, x+\Delta x}^{\mathcal{S}^{\prime}} \chi(x+\Delta x)\right),
\end{aligned}
$$

где $\mathcal{S}$ и $\mathcal{S}^{\prime}$ - это кратчайшие пути, ведущие в точку $x^{\prime}$ из точек $x$ и $x+\Delta x$ соответственно, причем сама точка $x^{\prime}$ лежит на линии, которая соединяет кварк и антикварк, находящиеся в точках $x$ и $x+\Delta x$.

Уравнение для спектра масс в этой модели теперь можно получить методом, аналогичным использованному для $(2+1)$-мерного случая [1]. Если ввести перенормировку безразмерной константы связи $g$,

$$
g^{2}=\bar{g}^{2} \frac{a}{L_{\mathrm{had}}},
$$

и использовать дополнительно к (19) новые обозначения

$$
r^{2}=\frac{a^{2}\left(l_{1}^{2}+l_{2}^{2}\right)}{L_{\mathrm{had}}^{2}}=\frac{x_{1}^{2}+x_{2}^{2}}{L_{\mathrm{had}}^{2}}, \quad \kappa=\frac{\alpha \bar{g}^{2}}{4} \frac{1}{2}\left(N-\frac{1}{N}\right), \quad \tau=\frac{\bar{g}^{2}}{2 \pi} \frac{1}{2}\left(N-\frac{1}{N}\right),
$$

то после перехода к пределу $L \rightarrow \infty, L \eta_{0}=$ const, $a \rightarrow 0$ спектральное уравнение можно записать следующим образом:

$$
\begin{aligned}
\lambda f(\xi, \mathbf{r})= & {\left[\kappa^{2} r^{2}+\left(\frac{1}{\xi}+\frac{1}{1-\xi}\right)\left(-\nabla^{2}+\bar{m}_{\mathrm{q}}^{2}\right)\right] f(\xi, \mathbf{r})-} \\
& -\tau \mathrm{P} \int_{0}^{1} d \xi^{\prime} \frac{f\left(\xi^{\prime}, \mathbf{r}\right)-f(\xi, \mathbf{r})}{\left(\xi^{\prime}-\xi\right)^{2}}
\end{aligned}
$$

Здесь $\nabla^{2}$ - это двухмерный оператор Лапласа, $\mathbf{r}$ - вектор в двумерном поперечном пространстве, а $r$, как и в $(2+1)$-мерном случае, - расстояние между кварком и антикварком. Таким образом, оказалось, что построение $(3+1)$-мерной модели из гамильтониана (21) в данном приближении полностью эквивалентно наиболее прямому обобщению спектрального уравнения $(20)$ на $(3+1)$-мерный случай.

Уравнение (27) можно легко обобщить на случай кварков разной массы $\bar{m}_{1,2}$, проведя замену

$$
\left(\frac{1}{\xi}+\frac{1}{1-\xi}\right) \bar{m}_{\mathrm{q}}^{2} \longrightarrow \frac{\bar{m}_{1}^{2}}{\xi}+\frac{\bar{m}_{2}^{2}}{1-\xi} .
$$

В принципе, это обобщение можно было сделать на уровне лагранжиана изначальной теории, с самого начала рассматривая кварки разной массы. В этом случае удобно воспользоваться параметризацией

$$
k=\frac{\bar{m}_{1}-\bar{m}_{2}}{\bar{m}_{1}+\bar{m}_{2}}, \quad \mu=\bar{m}_{1}+\bar{m}_{2}, \quad \bar{m}_{1}=\frac{\mu}{2}(1+k), \quad \bar{m}_{2}=\frac{\mu}{2}(1-k)
$$

и совершить замену переменных

$$
\omega=2 \xi-1, \quad-1 \leqslant \omega \leqslant 1, \quad f(\xi)=f\left(\frac{\omega+1}{2}\right)=\varphi(\omega) .
$$


В результате уравнение (27) принимает следующий вид:

$$
\begin{aligned}
\lambda \varphi(\omega, \mathbf{r})= & {\left[-\frac{4}{1-\omega^{2}} \nabla^{2}+\kappa^{2} r^{2}\right] \varphi(\omega, \mathbf{r})+\left[1+\frac{(k-\omega)^{2}}{1-\omega^{2}}\right] \mu^{2} \varphi(\omega, \mathbf{r})+} \\
& +2 \tau \mathrm{P} \int_{-1}^{1} d \omega^{\prime} \frac{\varphi(\omega, \mathbf{r})-\varphi\left(\omega^{\prime}, \mathbf{r}\right)}{\left(\omega-\omega^{\prime}\right)^{2}} .
\end{aligned}
$$

Поскольку это уравнение обладает вращательной симметрией в поперечном пространстве, целесообразно ввести полярную систему координат, в которой лапласиан имеет вид

$$
\nabla^{2}=\frac{\partial^{2}}{\partial r^{2}}+\frac{1}{r} \frac{\partial}{\partial r}+\frac{1}{r^{2}} \frac{\partial^{2}}{\partial \theta^{2}}
$$

В этой системе координат можно разделить переменные с помощью факторизации

$$
\varphi(\omega, \mathbf{r})=W(\omega, r) Q(\theta)
$$

и подстановки

$$
\frac{\partial^{2}}{\partial \theta^{2}} Q(\theta)=-L_{z}^{2} Q(\theta),
$$

которая элементарно разрешается относительно $Q(\theta)$ :

$$
Q(\theta)=e^{i L_{z} \theta}, \quad L_{z}=0, \pm 1, \pm 2, \ldots .
$$

В результате этих преобразований мы получаем окончательное уравнение для спектра масс в нашей модели, которое записывается следующим образом:

$$
\begin{aligned}
\lambda W(\omega, r)= & \frac{4}{1-\omega^{2}}\left[-\frac{\partial^{2}}{\partial r^{2}}-\frac{1}{r} \frac{\partial}{\partial r}+\frac{1}{r^{2}} L_{z}^{2}+(k-\omega)^{2}\left(\frac{\mu}{2}\right)^{2}\right] W(\omega, r)+ \\
& +\left(\mu^{2}+\kappa^{2} r^{2}\right) W(\omega, r)+2 \tau \mathrm{P} \int_{-1}^{1} d \omega^{\prime} \frac{W(\omega, r)-W\left(\omega^{\prime}, r\right)}{\left(\omega-\omega^{\prime}\right)^{2}}
\end{aligned}
$$

Асимптотика этого уравнения при $r \rightarrow 0$ имеет вид

$$
W(\omega, r) \underset{r \rightarrow 0}{\propto} r^{\left|L_{z}\right|} .
$$

При $L_{z}=0$ функция $\left.W(\omega, r)\right|_{r=0}$ пропорциональна некоторой константе и удовлетворяет граничным условиям Неймана. При $L_{z} \neq 0$ функция $\left.W(\omega, r)\right|_{r=0}$ равна нулю и, следовательно, удовлетворяет граничным условиям Дирихле. В дополнение к этому из анализа уравнения 'т Хоофта [15] следует, что $W(\omega, r)$ должна удовлетворять определенным граничным условиям по переменной $\omega$ при $\omega= \pm 1$. Таким образом, полный набор граничных условий, необходимый для решения задачи на собственные значения уравнения (36), записывается как

$$
\begin{gathered}
\partial_{r} W(\omega, 0)=0 \text { для } L_{z}=0, \quad W(\omega, 0)=0 \text { для } L_{z} \neq 0, \\
W(\omega, \infty)=W( \pm 1, r)=0 .
\end{gathered}
$$

Собственные значения и волновые функции уравнения (36) не поддаются аналитическому описанию, но могут быть получены численно с использованием указанных граничных условий. Это вычисление было нами проведено, и его детали кратко 


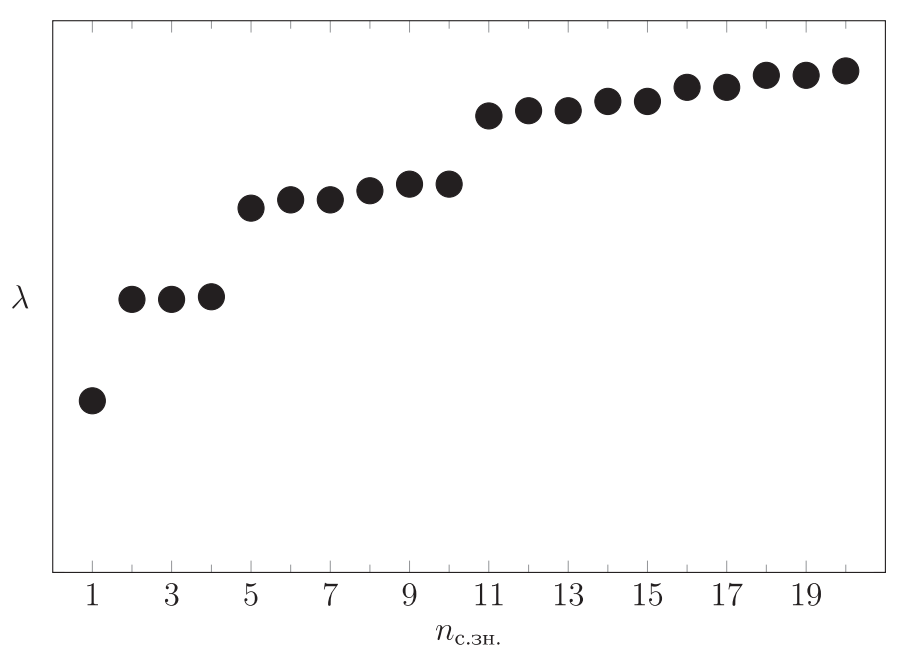

Рис. 2. Спектр уравнения (36) для некоторого набора параметров. Здесь

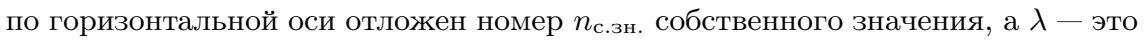
безразмерная величина квадрата массы в произвольных единицах.

приведены в приложении. На рис. 2 изображен спектр нашей модели при некотором наборе параметров. Качественно этот спектр напоминает спектр гармонического осциллятора в трех измерениях. Примечательной особенностью нашей модели является возможность появления вырожденных эквидистантных уровней энергии, которые соответствуют линейным траекториям Редже. Это важный результат, поскольку в экспериментальном спектре мезонов действительно наблюдается значительное вырождение [16]-[19]. Волновые функции, полученные в рамках нашего подхода, теоретически можно использовать для расчета постоянных распада [20] и партонных распределений [21], [22].

\section{4. ЗАКЛЮЧЕНИЕ И ОБСУЖДЕНИЕ}

Настоящая работа посвящена построению эффективной модели кварк-антикваркового взаимодействия. В работе [14] был получен эффективный гамильтониан в формализме квантования на СФ с динамическими нулевыми модами и решеточной регуляризацией в поперечном пространстве. В нашей модели рассматривается только проекция этого гамильтониана на пространство состояний с одним кварком и одним антикварком, которые соединены калибровочно-инвариантным образом в поперечном пространстве “струной” из матриц, отвечающих нулевой моде глюонного поля. С использованием такого приближения в работе [1] было получено уравнение для спектра масс в $(2+1)$-мерной модели. В настоящей работе представлено обобщение этого результата на физически более интересный $(3+1)$-мерный случай. Переход к $(3+1)$-мерной модели соответствует переходу от одномерной решетки в поперечном пространстве к двумерной. Это значительно усложняет анализ возможных состояний. Для упрощения задачи мы предполагаем, что кварк и антикварк соединены в этом поперечном пространстве преимущественно по кратчайшему пути. Также мы совершаем нелокальную модификацию четырехфермионного члена в эффективном 
гамильтониане аналогично $(2+1)$-мерному случаю [1], обобщая ее на $3+1$ измерение. В этом приближении удается получить уравнение (36) для спектра масс. Это спектральное уравнение имеет тот же вид, что и уравнение для $(2+1)$-мерного случая, с точностью до замены соответствующих одномерных величин на двумерные.

Поскольку собственные значения и волновые функции уравнения (36) не удается получить аналитически, для обсуждения их качественных свойств мы приводим численное решение при некотором наборе параметров. Полученный спектр изображен на рис. 2, а детали вычисления приведены в приложении. Качественно этот спектр напоминает спектр гармонического осциллятора в трех измерениях, для которого характерно наличие главного квантового числа, ответственного за уровень энергии. В работах [23]-[25] приведена классификация мезонов по радиальным и орбитальным квантовым числам и показано, что вырождение хорошо описывается эмпирической формулой для квадрата массы

$$
M^{2}=a\left(l+n_{\mathrm{r}}\right)+b,
$$

где $l$ - это квантовое число углового момента, $n_{\mathrm{r}}$ нумерует радиальные возбуждения, параметры $a \approx 1.1$ Гэ $\mathrm{B}^{2}$ и $b \approx 0.7$ Гэ $\mathrm{B}^{2}$ определяются из экспериментальных данных, а комбинация $l+n_{\mathrm{r}}$ выступает в роли главного квантового числа.

В уравнении (36), задающем спектр масс нашей модели, виден вклад, подобный гармоническому осциллятору в поперечном пространстве, а также продольная часть, которая выглядит как уравнение 'т Хоофта [15]. Известно, что спектр уравнения 'т Хоофта для квадрата массы мезона асимптотически линеен и в этом смысле схож с линейным спектром энергии гармонического осциллятора. Это, по-видимому, объясняет, почему при определенном выборе параметров спектр нашей модели является вырожденным и эквидистантным.

В нашей модели присутствуют три безразмерных параметра: обезразмеренная масса кварка $\bar{m}_{\mathrm{q}}=m_{\mathrm{q}} L_{\mathrm{had}}$, константа связи $g$ и параметр $\alpha$, который связывает величину $L \eta_{0}$ с адронным масштабом: $L_{\mathrm{had}}=\alpha L \eta_{0}$. Также существенно, что мы ввели в четырехфермионный член нелокальность на масштабах порядка размера адрона $L_{\text {had }}$. Интересен тот факт, что параметры, отвечающие вырождению спектра, соответствуют $\alpha \sim 1$, а следовательно, $L \eta_{0}$ имеет порядок $L_{\mathrm{had}}$.

В работе [26] была предпринята попытка моделировать экспериментальный спектр мезонов с присущим ему вырождением с помощью потенциальных кварковых моделей. Автор исследует вопрос о том, возможно ли найти такой потенциал, который приводит к появлению главного квантового числа, подобного тому, что возникает в модели с кулоновским потенциалом или с потенциалом гармонического осциллятора. Используя обобщение теоремы Бертрана, он приходит к выводу о том, что потенциальные модели сталкиваются с принципиальными трудностями при описании вырождения в экспериментальном спектре мезонов. Наша окончательная модель, представленная уравнением (36), является потенциальной только в поперечном направлении, а в продольном аналогична уравнению 'т Хоофта двумерной КХД. Это, в принципе, позволяет обойти ограничения, связанные с потенциальными моделями.

Отметим, что задача вычисления массы связанных состояний кварка и антикварка, в которой возникает аналог уравнения 'т Хоофта, рассматривалась также в работе [27]. При этом использовалась модель, которая получается из $(3+1)$-мерной КХД путем редукции $(3+1)$-мерного пространства к $(1+1)$-мерному за счет 
компактификации поперечных измерений и проекции на подпространство нулевых мод по соответствующим координатам. В этой работе показано, что за счет учета нулевых мод поперечных компонент глюонного поля появляется инфракрасная неустойчивость решения уравнения 'т Хоофта из двумерной КХД и, соответственно, низшая часть спектра может стать тахионной. Авторами работы [27] было найдено непертурбативное решение для динамической массы кварка, которое разрешает эту неустойчивость и делает спектр масс вещественным. В киральном пределе низшее состояние в спектре мезонов этой модели является безмассовым и интерпретируется как пион.

Существуют также попытки построения модели для описания спектра мезонов из голографических соображений с использованием формализма СФ [28], [29]. Отправной точкой для них является полученное из рассмотрения дуальности $\mathrm{AdS}_{5} / \mathrm{QCD}$ эффективное уравнение типа Шредингера на СФ для волновой функции мезона. В него входит потенциал в поперечном пространстве, который выбирается исходя из различных голографических моделей. Одна из таких моделей приводит к спектру гармонического осциллятора для квадрата массы мезона. Предполагается, что волновую функцию можно представить как произведение поперечной и продольной части, а продольная часть выбирается либо произвольным образом, либо в виде волновой функции, отвечающей низшему значению в спектре уравнения 'т Хоофта [29]. В отличие от этого подхода, в нашей модели как поперечный потенциал, так и уравнение 'т Хоофта появляются более или менее физически естественным образом из обычной КХД благодаря рассмотрению нулевой моды глюонного поля как независимой динамической переменной и нелокальной модификации взаимодействия фермионных токов. Тем самым, в отличие от указанных голографических моделей, в нашем спектре могут присутствовать возбуждения в том числе и по продольной координате, а не только низшее состояние. Также заметим, что в нашей модели можно "отключить" продольную часть, выбрав параметры так, что модель будет подобна указанным выше голографическим моделям со спектром двухмерного гармонического осциллятора.

Полученные нами волновые функции теоретически можно использовать для расчета постоянных распада [20] и партонных распределений [21], [22].

\section{ПРИЛОЖЕНИЕ}

Чтобы решить уравнение (36) численно, мы ограничиваем радиальное пространство условием $0 \leqslant r \leqslant r_{0}$ и вводим решетку с шагом $a_{r}$ :

$$
r \rightarrow r_{l}=r_{0} a_{r} l, \quad l=0,1, \ldots, \frac{1}{a_{r}} .
$$

Физически это означает, что мы рассматриваем область поперечного пространства, имеющую радиус $r_{0} L_{\text {had }}$. Далее мы дискретизуем относительный импульс на интервале $-1 \leqslant \omega \leqslant 1$ с шагом $a_{\omega}$ :

$$
\omega \rightarrow \omega_{m}=a_{\omega} m-1, \quad m=0,1, \ldots, \frac{2}{a_{\omega}},
$$


и вводим дискретный аналог волновой функции $W(\omega, r) \rightarrow W_{m, l}$. Дискретизация уравнения (36) завершается введением дискретных аналогов производных и интеграла:

$$
\begin{gathered}
\frac{\partial^{2}}{\partial r^{2}} W(\omega, r) \rightarrow \frac{W_{m, l-1}-2 W_{m, l}+W_{m, l+1}}{a_{r}^{2}}, \\
\frac{\partial}{\partial r} W(\omega, r) \rightarrow \frac{-W_{m, l-1}+W_{m, l+1}}{2 a_{r}}, \\
\mathrm{P} \int_{-1}^{1} d \omega^{\prime} \frac{W(\omega, r)-W\left(\omega^{\prime}, r\right)}{\left(\omega-\omega^{\prime}\right)^{2}} \rightarrow \frac{1}{a_{\omega}} \sum_{\substack{n=0 \\
n \neq m}}^{2 / a_{\omega}} \frac{W_{m, l}-W_{n, l}}{(m-n)^{2}} .
\end{gathered}
$$

Далее, если каким-либо единым образом пронумеровать все независимые переменные как $W_{m, l} \rightarrow \widehat{W}_{i}$, то можно прийти к матричному уравнению

$$
\Lambda_{i j} \widehat{W}_{j}=\lambda \widehat{W}_{j}
$$

Его решение с учетом граничных условий (38) позволяет найти собственные значения и волновые функции.

Благодарности. Авторы благодарят С. С. Афонина за многочисленные и плодотворные обсуждения.

\section{Список литературы}

[1] Р. А. Зубов, Е. В. Прохватилов, М. Ю. Малышев, "Предельный переход к световому фронту в квантовой хромодинамике и кварк-антикварковое приближение", ТМФ, 184:3 (2015), 456-464.

[2] P. A. M. Dirac, "Forms of relativistic dynamics", Rev. Modern Phys., 21:3 (1949), 392-398.

[3] M. Burkardt, A. Langnau, "Hamiltonian formulation of $(2+1)$-dimensional QED on the light cone", Phys. Rev. D, 44:4 (1991), 1187-1197.

[4] M. Burkardt, A. Langnau, "Rotational invariance in light-cone quantization", Phys. Rev. D, 44:12 (1991), 3857-3867.

[5] С. А. Пастон, В. А. Франке, “Сравнение квантово-полевой теории возмущений на световом фронте и в лоренцевых координатах", ТМФ, 112:3 (1997), 399-416, arXiv: hep-th/9901110.

[6] V.A. Franke, Yu. V. Novozhilov, S. A. Paston, E. V. Prokhvatilov, "Quantization of field theory on the light front", Focus on Quantum Field Theory, ed. O. Kovras, Nova Sci. Publ., New York, 2005, 23-81, arXiv: hep-th/0404031.

[7] С. А. Пастон, Е.В. Прохватилов, В.А. Франке, "К построению гамильтониана КХД в координатах светового фронта", ТМФ, 120:3 (1999), 417-437, arXiv: hep-th/0002062.

[8] Е. В. Прохватилов, В. А. Франке, "Предельный переход к светоподобным координатам в теории поля и КХД-гамильтониан", ЯФ, 49:4 (1989), 1109-1117.

[9] V. A. Franke, Yu. V. Novozhilov, E. V. Prokhvatilov, "On the light-cone formulation of classical non-abelian gauge theory", Lett. Math. Phys., 5:3 (1981), 239-245.

[10] V. A. Franke, Yu. V. Novozhilov, E. V. Prokhvatilov, "On the light-cone quantization of nonabelian gauge theory", Lett. Math. Phys., 5:5 (1981), 437-444.

[11] E. V. Prokhvatilov, H. W. L. Naus, H.-J. Pirner, "Effective light-front quantization of scalar field theories and two-dimensional electrodynamics", Phys. Rev. D, 51:6 (1995), 2933-2942. 
[12] Е.-М. Ильгенфриц, С.А. Пастон, Г. Ю. Пирнер, Е. В. Прохватилов, В. А. Франке, "Квантовые поля на световом фронте, формулировка в координатах, близких к световому фронту, решеточное приближение", ТМФ, 148:1 (2006), 89-101, arXiv: hep-th/0610020.

[13] Е. В. Прохватилов, В. А. Франке, "Приближенное описание КХД конденсатов в светоподобных координатах", ЯФ, 47:3 (1988), 882-883.

[14] М.Ю.Малышев, Е.В.Прохватилов, "Квантовая хромодинамика на световом фронте с нулевыми модами, моделирующими вакуум", ТМФ, 169:2 (2011), 272-284.

[15] G. 't Hooft, "A two-dimensional model for mesons", Nucl. Phys. B, 75:3 (1974), 461-470.

[16] D. V. Bugg, "Four sorts of meson", Phys. Rep., 397:5 (2004), 257-358.

[17] S.S. Afonin, "Experimental indication on chiral symmetry restoration in meson spectrum", Phys. Lett. B, 639:3-4 (2006), 258-262, arXiv: hep-ph/0603166.

[18] S.S. Afonin, "Light meson spectrum and classical symmetries of QCD", Eur. Phys. J. A, 29:3 (2006), 327-335, arXiv: hep-ph/0606310.

[19] M. Shifman, A. Vainshtein, "Highly excited mesons, linear Regge trajectories, and the pattern of the chiral symmetry realization", Phys. Rev. D, 77:3 (2008), 034002, 15 pp., arXiv: 0710.0863.

[20] S. J. Brodsky, T. Huang, G. P. Lepage, "Hadronic wave functions and high momentum transfer interactions in quantum chromodynamics", Particles and Fields 2 (Summer institute, Banff, Canada, August 16-27, 1981), eds. A. Z. Capri, A. N. Kamal, Plenum Press, New York, 1983, 143-199.

[21] A. Vega, I. Schmidt, T. Branz, T. Gutsche, V. E. Lyubovitskij, "Meson wave function from holographic models", Phys. Rev. D, 80:5 (2009), 055014, 8 pp., arXiv: 0906.1220.

[22] A. V. Radyushkin, "Nonforward parton densities and soft mechanism for form factors and wide-angle Compton scattering in QCD", Phys. Rev. D, 58:11 (1998), 114008, 12 pp., arXiv: hep-ph/9803316.

[23] S.S. Afonin, "Towards understanding broad degeneracy in non-strange mesons", Modern Phys. Lett. A, 22:19 (2007), 1359-1371, arXiv: hep-ph/0701089.

[24] S.S. Afonin, "Properties of possible new unflavored mesons below $2.4 \mathrm{GeV",} \mathrm{Phys.} \mathrm{Rev.} \mathrm{C,}$ 76:1 (2007), 015202, 5 pp., arXiv: 0707.0824.

[25] S. S. Afonin, "Hydrogen like classification for light nonstrange mesons", Internat. J. Modern Phys. A, 23:25 (2008), 4205-4217, arXiv: 0709.4444.

[26] P. Bicudo, "Large degeneracy of excited hadrons and quark models", Phys. Rev. D, 76:9 (2007), 094005, 9 pp., arXiv: hep-ph/0703114.

[27] J. Alfaro, P. Labraña, A. A. Andrianov, "Extended $\mathrm{QCD}_{2}$ from dimensional projection of QCD4", JHEP, 07 (2004), 067, 16 pp., arXiv: hep-th/0309110.

[28] S. J. Brodsky, G.F. de Téramond, "Hadronic spectra and light-front wave functions in holographic QCD", Phys. Rev. Lett., 96:20 (2006), 201601, 4 pp., arXiv: hep-ph/0602252.

[29] S.S. Chabysheva, J.R. Hiller, "Dynamical model for longitudinal wave functions in light-front holographic QCD", Ann. Phys., 337 (2013), 143-152, arXiv: 1207.7128. 